\title{
The Florida State Twin Registry: Research Aims and Design
}

\author{
Jeanette E. Taylor, Lisa M. James, Mark D. Reeves, and Leonardo Bobadilla \\ Department of Psychology, Florida State University, Tallahassee, Florida, United States of America
}

\begin{abstract}
R elatively little is known about the relationship of most personality disorders to executive cognitive functioning despite their associations with frontal cortex activity. Research on genetic influence is lacking for most personality disorders, and research on genetic influences associated with executive cognitive functioning is sparse and mixed. The Florida State Twin Registry was created to conduct a pilot twin study aimed at examining genetic influence on personality disorders and executive cognitive functioning. Measures included structured clinical interviews for symptoms and diagnoses of personality disorders (borderline, histrionic, narcissistic, antisocial, obsessive-compulsive, avoidant, and dependent), depression, substance abuse/dependence, anxiety disorders, and eating disorders. The Wisconsin Card Sorting Test and the Stroop ColorWord Test were administered to assess executive cognitive functioning. Self-report questionnaires were included to assess maladaptive personality traits. Data sharing and future directions for growing the Florida State Twin Registry are discussed.
\end{abstract}

Personality disorders (PDs) reflect maladaptive cognitive and behavior patterns that cause significant impairment or subjective distress (American Psychiatric Association, 1994). Personality disorders present special challenges to clinicians because they are often comorbid with and can adversely affect the treatment of other mental disorders (e.g., Target, 1998). A clearer understanding of the etiological factors associated with PDs could contribute to improvements in their classification and perhaps to efforts at developing effective treatments. The etiology of a PD is likely a complex mix of biological (e.g., genetic), psychological (e.g., cognitive), and environmental factors. Although genetic influence has been investigated in certain PDs, most have received little attention in this regard (Dahl, 1993).

Cognitive functions of the frontal cortex include working memory, forethought, and planning. The frontal lobes have historically been associated with personality (Stuss \& Alexander, 2000), and yet relatively little work has been done investigating functioning of the frontal lobes as it relates to personality pathology. The existing literature suggests an association between executive cognitive functioning and antisocial (Mazas et al., 2000), borderline (Sprock et al., 2000), and schizotypal (Diforio et al., 2000) PDs, but it reveals nothing about the association between executive cognitive functioning and other PDs.

Evidence for genetic influence on PD comes indirectly from reports by Livesley and his colleagues on a large volunteer twin sample. Livesley's Dimensional Assessment of Personality Problems - Differential Questionnaire (DAPP-DQ) assesses a broad range of PD features (18 primary factors) using a personality trait model (Livesley \& Jackson, in press). The primary factors are comprised of more specific facets and the 18 primary factors load onto four higher order factors reflecting a hierarchical structure for PD traits. Twin data suggest that the 18 primary factors and higher order dimensions are influenced by genetic and nonshared environmental factors (Jang, Livesley, Vernon, \& Jackson, 1996; Livesley et al., 1992) and genetic factors account for unique variance in the 18 primary factors once the variance from the higher order factors is partialed out (Livesley et al., 1998). Further analyses of the DAPP data have shown that genetic factors may increase in their influence on PD traits with age (Jang, Livesley, \& Vernon, 1996), and most of the primary factors evidence sex-specific genetic effects (Jang et al., 1998). Finally, Jang and Livesley (1999) found that the Big Five personality traits had a high genetic correlation with the DAPP primary factors, indicating that normal personality traits and PD traits share a genetic etiology (as would be expected if PDs can be accounted for by dimensional models of normal personality).

Reviews of the literature on the genetics of PD conclude that data on most PDs are lacking (Dahl, 1993; Nigg \& Goldsmith, 1994). Antisocial PD has received a lot of attention and the evidence suggests that genetic factors are associated with about $30 \%$ of the variance in that diagnosis. Familial aggregation of borderline PD indicates possible genetic transmission for that PD (Dahl, 1993). Schizotypal PD has been

\section{Received 8 July, 2006; accepted 15 July, 2006.}

Address for correspondence: Jeanette Taylor, Department of Psychology, 209 Eppes Hall, Florida State University, Tallahassee, FL 32306-1270,USA.E-mail: taylor@psy.fsu.edu 
linked in family studies to schizophrenia (which is heritable) and a single twin study of schizotypal PD found direct evidence of genetic influence (Dahl, 1993). All of the other PDs have either received no attention in behavioral genetic research or have been the focus of only family studies (Dahl, 1993). Torgersen et al. (2000) provided the first and only published twin study of the full range of PD diagnoses as assessed via structured interview. Genetic effects accounted for 28 to $77 \%$ of the variance in PD diagnoses with the remaining variance associated with nonshared environmental effects. Similarly, genetic factors accounted for $1 / 3$ to $3 / 5$ of the variance of diagnoses within each DSM cluster. Torgersen et al. (2000) did not report on genetic and environmental effects on the covariation of PDs.

Results from studies examining genetic influence on executive cognitive abilities are mixed. A study of mother's ratings of executive cognitive functions in young twins (mean age 8 years) found that $79 \%$ of the variance was associated with additive genetic effects and the remaining variance was accounted for by nonshared environmental factors (Coolidge et al., 2000). Two other twin studies failed to find genetic influence on performance on the Wisconsin Card Sorting Test (WCST; Campana et al., 1996, Nicole \& Del Miglio, 1997). However, both were based on very small twin samples (less than 15 pairs of monozygotic [MZ] and dizygotic [DZ] twins). More recently, Anokhin et al. (2003) found that some scores from the WCST showed moderate genetic influence whereas other scores showed no genetic influence in a relatively small sample of female twins. The literature suggests that executive cognitive functioning is associated with features of certain PDs (e.g., antisocial), but the etiological underpinnings of that association is not clear. It may be that executive cognitive functioning provides a common pathway to the expression of features of certain PDs (e.g., those marked by impulsivity).

The Florida State Twin Registry (FSTR) was created in the course of recruiting twins into a paid research study aimed at examining genetic and environmental influences on PDs, executive cognitive functions, and the covariation of those variables.

\section{Materials and methods \\ Registry Membership}

Recruitment for the FSTR began in 2002 and it currently contains 153 twin pairs: $98 \mathrm{MZ}$ (29 male); 34 same-sex DZ (6 male); 17 opposite-sex; and 4 triplet sets. Zygosity is unknown for an additional 57 pairs who were unable to be reached for follow-up after their initial contact. Twins in the registry range in age from 7 to 84 years. The ethnic composition of the registry reflects that of Florida State University and the greater Tallahassee area: $76 \%$ white, $16 \%$ AfricanAmerican, 4\% Hispanic, and 4\% other or mixed race. Many of the twins were ascertained using public information from the Florida State University registrar's office. Specifically, at the beginning of each academic semester, the registrar provided the authors with a list of people within the university (primarily undergraduate students) who matched on last name, date of birth, and city of birth, and this list comprised potential twin pairs. The match included current and permanent postal addresses and an e-mail address. A mailing was sent to people on the match list that included a cover letter explaining our interest in twins as participants in a paid research study and requesting that they complete a reply form and return it in the self-addressed stamped envelope. The person was able to indicate on the reply form whether they were a twin and, if so, provide current contact information (as an update to what was received from the registrar) and indicate whether they were interested in (1) being included in the FSTR, and (2) learning more about the current paid research study. One hundred and twenty twin pairs or triplet sets currently in the registry were ascertained via this method. The remaining members of the registry were recruited via newspaper advertisements for the paid research study.

\section{Research Study Sample}

Twins were eligible for the research study if they were at least 18 years old and had no self-reported history of head injury or neurological disorder. Sixteen twin pairs were disqualified based on one of these exclusion criteria. Of the remaining twins in the registry, $81 \mathrm{MZ}$ pairs (22 male) and 27 same-sex DZ pairs (4 male) were successfully recruited into the study. Twins in the study ranged in age from 18 to 84 years old $(M=$ 23.76; $S D=11.68)$. Seventy-seven per cent were white; $16 \%$ were African-American; $5 \%$ were Hispanic; and $3 \%$ were other or mixed race.

\section{Zygosity Diagnosis}

Zygosity was determined using a 5 -item self-report questionnaire completed by the twins, and by a rating of twin similarity on natural hair color, natural eye color, ear shape and ear lobe attachment completed by trained research staff. At the time of the similarity rating, a close-up digital photograph was taken of an eye and an ear of each twin for use in resolving discrepancies between the similarity rating and self-reported zygosity questionnaire. Discrepancies in twin and staff ratings occurred in $8 \%$ of the sample. Most discrepancies could be resolved by examining the digital photographs and those that could not be resolved were coded according to the twin report on the zygosity questionnaire.

\section{Research Study Procedure.}

Twins reported to a laboratory on the Florida State University campus for a 3.25 hour study. Informed consent was obtained by an undergraduate research assistant prior to beginning any procedures; the consent form included explicit consent for the co-twin to be interviewed regarding the twin's personality. The undergraduate research assistant conducted the twin similarity rating and then the twins were separated to 
independently complete the remaining procedures. Cognitive functioning measures were administered first followed by self-reports. Finally, twins were independently interviewed for symptoms of personality and other psychological disorders by a clinical psychology graduate student with advanced training and experience with clinical interviewing. At the end of the study, each twin was paid \$30 USD for participating.

\section{Research Study Measures}

Table 1 presents a list of the measures included in the research study from its start. Based on an examination of preliminary data from the clinical interviews for PDs, a protocol change was made in 2004 that eliminated the assessment of symptoms of schizoid, schizotypal, and paranoid PDs (which were rare) in favor of including additional cognitive tasks: the Iowa Gambling Task and the Shipley Institute of Living scale. As such, those measures were assessed on only part $(47 \%)$ of the sample.

\section{Diagnostic Variables}

Following procedures used on the Minnesota Twin Family Study, symptoms of psychological disorders were assigned during case conferences in which at least two clinical psychology graduate students examined all available information (including, when necessary, tape recordings of the clinical interviews) and came to a consensus agreement regarding each symptom (absent, subthreshold present, or threshold present). For the PDs, the consensus team considered not only the twin's own report on the Structured Interview for DSM-IV Personality Disorders (SIDP-IV; Pfohl et al., 1995) but also the co-twin's collateral
SIDP-IV report. The SIDP-IV collateral report is based on a selected set of questions for each disorder and, therefore, is not appropriate for deriving independent symptom counts or diagnoses. Time limits on the length of the study protocol precluded an expansion of the SIDP-IV collateral report procedure to include assessment of all SIDP-IV questions. Moreover, the twin's own report was given priority when time ran short and, in some cases, the collateral twin report could not be obtained at all. In considering the information from the twin and the co-twin reports, the consensus team employed a best estimate strategy for symptom assignment in that a symptom could be endorsed by either informant, and reporter agreement was not required in order to assign the symptom as present.

\section{Data Management, Registry Access and Collaboration}

All data are stored electronically in tables within a single Microsoft Access database. The Access tables are easily read into SPSS or other statistical software packages where data tables for different measures can be easily merged. A procedure for providing outside researchers access to the registry members for recruitment into research studies has been established. Written requests for access to the registry are sent to Dr Jeanette Taylor (see correspondence information listed in this article). Registry access would be considered only for project protocols with documented Institutional Review Board approval. Dr Taylor would weigh requests for access against the need to not overburden registry members with research participation requests. Once a project has been approved, a recruitment statement that includes contact information for the outside researcher is provided to Dr Taylor who

\section{Table 1}

Description of Measures in the Florida State Twin Registry

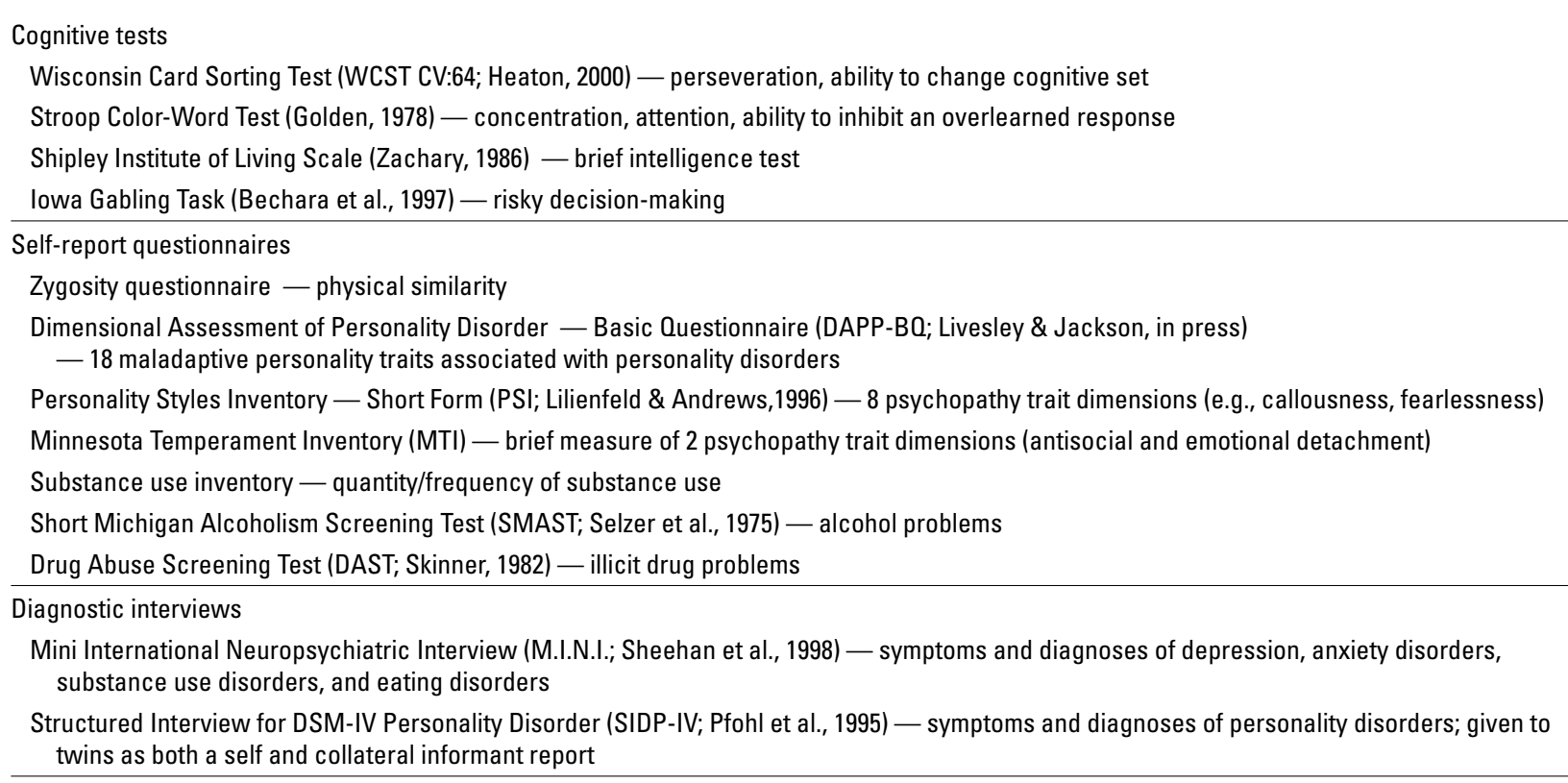


then contacts twins in the registry about the research opportunity being offered. The outside researcher is then responsible for any postal charges incurred. Twins interested in the research opportunity being offered then contact the outside researcher directly about participating in the research. Requests for collaborations are also sent to Dr Taylor. Collaborations could take many forms including requests for access to existing data (e.g., for examination in conjunction with similar data collected on another twin sample), collection of new data to compliment existing data, or joint collection of new data in multiple twin registries.

\section{Future Directions}

The FSTR is growing in a new direction in 2006 with the start of a multidisciplinary center grant funded by the National Institute of Child Health and Human Development aimed at examining reading disability. One of the center grant projects entails ascertainment of twins (using the matching criteria noted above) from the Florida's Progress Monitoring and Reporting Network (PMRN) that contains data on reading and achievement from 300,000 children in Kindergarten through fifth grade in underachieving schools in Florida. Children in the PMRN are racially diverse: approximately $62 \%$ are white and approximately $36 \%$ are African-American with the remaining children from other racial groups. About $20 \%$ of the children are of Hispanic ethnicity. Approximately 124,000 new children enter the database each year, and we hope to identify around 9000 twin pairs over the course of 5 years. Parents of identified twins will be asked for permission to include the twins in the FSTR. This expansion of the FSTR will allow for future data collection opportunities through follow-up assessments with the twins.

\section{$\overline{\text { Acknowledgment }}$}

This article was supported in part by NIMH grant MH067583.

\section{References}

American Psychiatric Association. (1994). Diagnostic and statistical manual of mental disorders (4th ed.). Washington, DC: Author.

Anokhin, A. P., Heath, A. C., \& Relano, A. (2003). Genetic influences on frontal brain function: WCST performance in twins. Cognitive Neuroscience and Neuropsychology, 14, 1975-1978.

Bechara, A., Damasio, H., Tranel, D., \& Damasio, A. R. (1997). Deciding advantageously before knowing the advantageous strategy. Science, 275, 1293-1294.

Campana, A., Macciardi, F., Gambini, O., \& Scarone, S. (1996). The Wisconsin Card Sorting Test (WCST) performance in normal subjects: A twin study. Neuropsychobiology, 34, 14-17.

Coolidge, F. L., Thede, L. L., \& Young, S. E. (2000). Heritability and the comorbidity of attention deficit hyperactivity disorder with behavioral disorders and executive function deficits: A preliminary investigation. Developmental Neuropsychology, 17, 273-287.

Dahl, A. A. (1993). The personality disorders: A critical review of family, twin, and adoption studies. Journal of Personality Disorders, (Suppl. 1), 86-99.

Diforio, D., Walker, E. F., \& Kestler, L. P. (2000). Executive functions in adolescents with schizotypal personality disorder. Schizophrenia Research, 42, 125-134.

Golden, C. J. (1978). Stroop Color and Word Test: A manual for clinical and experimental uses. Wood Dale, IL: Stoelting.

Heaton, R. K. (2000). WCST-64: Computer version for Windows - Research edition [Computer software]. Odessa, FL: Psychological Assessment Resources.

Jang, K. L., \& Livesley, W. J. (1999). Why do measures of normal and disordered personality correlate? A study of genetic comorbidity. Journal of Personality Disorders, 13, 10-17.

Jang, K. L., Livesley, W. J., \& Vernon, P. A. (1996). The genetic basis of personality at different ages: A crosssectional twin study. Personality and Individual Differences, 21, 299-301.

Jang, K. L., Livesley, W. J., \& Vernon, P. A. (1998). A twin study of genetic and environmental contributions to gender differences in traits delineating personality disorder. European Journal of Personality, 12, 331-344.

Jang, K. L., Livesley, W. J., Vernon, P. A., \& Jackson, D. N. (1996). Heritability of personality disorder traits: A twin study. Acta Psychiatrica Scandinavica, 94, 438-444.

Lilienfeld, S. O., \& Andrews, B. P. (1996). Development and preliminary validation of a self-report measure of psychopathic personality traits in noncriminal populations. Journal of Personality Assessment, 66, 488-524.

Livesley, W. J., \& Jackson, D. N. (in press). Manual for the dimensional assessment of personality pathology: Basic questionnaire. London: Research Psychologists Press.

Livesley, W. J., Jackson, D. N., \& Schroeder, M. L. (1992). Factorial structure of traits delineating personality disorders in clinical and general population samples. Journal of Abnormal Psychology, 101, 432-440.

Livesley, W. J., Jang, K. L., \& Vernon, P. A. (1998). Phenotypic and genetic structure of traits delineating personality disorders. Archives of General Psychiatry, 55, 941-948.

Mazas, C. A., Finn, P. R., \& Steinmetz, J. E. (2000). Decision-making biases, antisocial personality, and early-onset alcoholism. Alcoholism: Clinical and Experimental Research, 24, 1036-1040. 
Nicole, S., \& Del Miglio, C. (1997). Abstraction skillfulness in monozygotic and dizygotic twin pairs. Acta Geneticae Medicae et Gemellologiae, 46, 57-67.

Nigg, J. T., \& Goldsmith, H. H. (1994). Genetics of personality disorders: Perspectives from personality and psychopathology research. Psychological Bulletin, 115, 346-380.

Pfohl, B., Blum, N., \& Zimmerman, M. (1995). Structured Interview for DSM-IV Personality Disorders (SIDP-IV). Iowa City: University of Iowa.

Selzer M. L., Vinokur A., \& Van Rooijen, L. (1975). A self-administered Short Michigan Alcoholism Screening Test. Journal of Studies on Alcohol, 36, 117-126.

Sheehan, D. V., Lecrubier, Y., Harnett-Sheehan, K., Anorim, P., Janavs, J., Weiller, E., Hergueta, T., Baker, R., \& Dunbar, G. C., (1998). The MINI international neuropsychiatric interview (MINI): The development and validation of a structured diagnostic psychiatric interview. Journal of Clinical Psychiatry, 59(Suppl. 20), 22-33.
Skinner, H. A. (1982). The drug abuse screening test. Addictive Behaviors, 7, 363-371.

Sprock, J., Rader, T. J., Kendall, J. P., \& Yoder, C.Y. (2000). Neuropsychological functioning in patients with borderline personality disorder. Journal of Clinical Psychology, 56, 1587- 1600.

Stuss, D. T., \& Alexander, M. P. (2000). Executive functions and the frontal lobes: A conceptual view. Psychological Research, 63, 289-298.

Target, M. (1998). Outcome research on the psychosocial treatment of personality disorders. Bulletin of the Menninger Clinic, 62, 215-230.

Torgersen, S., Lygren, S., Oien, P. A., Skre, I., Onstad, S., Edvardsen, J., Tambs, K., \& Kringlen, E. (2000). A twin study of personality disorders. Comprehensive Psychiatry, 41, 416-425.

Zachary, R. A. (1986). Shipley Institute of Living Scale: Revised Manual. Los Angeles, CA: Western Psychological Services. 